\title{
Cribado tuberculínico; prevención de la tuberculosis
}

\author{
J. Pericas Bosch ${ }^{\mathrm{a}}$ y Grupo Prevlnfad/PAPPS Infancia y Adolescencia ${ }^{b}$ \\ aPediatra. CAP La Mina. Sant Adrià de Besòs, Barcelona. España. \\ ${ }^{b}$ Grupo PrevInfad (AEPap): FJ. Sánchez Ruiz-Cabello, FJ. Soriano Faura,J. Colomer Revuelta, \\ O. Cortés Rico, MJ. Esparza Olcina, J. Galbe Sánchez Ventura, J. García Aguado, A. Martínez Rubio, \\ M. Merino Moína, CR. Pallás Alonso, J. Pericas Bosch.
}

\begin{abstract}
Resumen
El cribado tuberculínico se utiliza para identificar, evaluar y poder tratar a las personas que tienen mayor riesgo de padecer una infección tuberculosa latente o de desarrollar la enfermedad tuberculosa una vez infectados por el M. tuberculosis. Se revisan los criterios, las indicaciones y la metodología del cribado tuberculínico para la detección de la infección tuberculosa y otras medidas disponibles en Atención Primaria para la prevención de la tuberculosis en la infancia y adolescencia, incluyendo el estudio de contactos de personas con infección tuberculosa y el tratamiento de la exposición a la tuberculosis y de la tuberculosis latente.
\end{abstract}

Palabras clave: Tuberculosis. Tuberculosis latente. Prueba de la tuberculina.

Tuberculin screening; tuberculosis prevention

Abstract

Tuberculin skin testing is used to identify, evaluate, and treat people who are at higher risk for latent tuberculosis infection or for developing tuberculosis disease after the infection by M. tuberculosis. We review the criteria, indications and methodology of tuberculin skin testing for the detection of tuberculosis infection and other measures available in primary care for the prevention of tuberculosis in childhood and adolescence, including the study of contacts of people with tuberculosis infection and the treatment of exposure to tuberculosis and of latent tuberculosis infection.

Key words: Tuberculosis. Latent tuberculosis. Tuberculin skin testing.

\section{Epidemiología}

A pesar de la disponibilidad desde hace décadas de tratamientos altamente eficaces, la tuberculosis (TBC) constituye un importante problema de salud pública en países en desarrollo y en bolsas de po- breza o marginación de los países desarrollados. La Organización Mundial de la Salud (OMS) estima que un tercio de la población mundial (2000 millones de personas) está infectada, se producen casi nueve millones de nuevos casos cada

Juan Pericas Bosch, jpericas@telefonica.net

Los autores declaran no presentar conflictos de intereses en relación con la preparación y publicación de este artículo. 
año y mueren 1,5 millones de personas anualmente por $\mathrm{TBC}^{1}$. Se consideran países con alta endemia aquellos con tasas de TBC de 100/100 000 habitantes 0 más. En España, la incidencia de infección tuberculosa es de 18/100 000, según el Ministerio de Sanidad y Consumo $(2005)^{2}$ y se estima una prevalencia del 1\% a los 6-7 años y del 3\% a los 13-14 años. La OMS estima para España una incidencia de 27/100 000. La media en países desarrollados es inferior a 10/100 000 .

La infección tuberculosa se había ido limitando a sujetos de edad avanzada y grupos marginales con menor facilidad para difundirla entre la población general, pero la inmigración procedente de países con alta prevalencia de TBC y la extensión de la infección por VIH han incrementado los casos de TBC en población joven, socialmente más activa y por ello más capaz de contagiar a nuevos individuos. Los inmigrantes procedentes de países en desarrollo y los infectados por VIH constituyen dos grupos que carecen a menudo de una atención sanitaria adecuada, en especial en sus aspectos preventivos.

La infección en niños suele depender del contacto y exposición prolongada con un adulto con enfermedad activa, bacilífero. En nuestro medio, el $40 \%$ de los contagios se produce antes de los cuatro años; el $80 \%$, antes de los 15, y el $95 \%$, antes de los 25 . El riesgo de desarrollar la enfermedad después de la primoinfección es de hasta un 10-15\%, máximo durante el año que sigue a la infección y aún importante durante los primeros cinco años. Cuanto menor es la edad del paciente cuando resulta infectado, más probable es que desarrolle la enfermedad. El $40-50 \%$ de los menores de un año, el $24 \%$ de los niños de uno a cinco años y el $15 \%$ de los adolescentes van a desarrollarla en los 1-2 años después de la infección. También cuanto menores son los pacientes más frecuentes resultan las formas extrapulmonares.

En el texto utilizaremos repetidamente estos tres conceptos epidemiológicos:

- Exposición a TBC sin evidencia de infección: contacto reciente, sustancial, con enfermo de TBC, asintomático, con derivado proteico purificado (PPD) negativo y radiología de tórax normal.

- Infección tuberculosa latente: contacto con el bacilo tuberculoso, demostrado por un PPD positivo, sin evidencia de enfermedad clínica.

- Enfermedad tuberculosa: infección demostrada por PPD y/o bacteriología positiva, junto a signos o síntomas clínicos o radiológicos diagnosticables. 
Detección de la infección tuberculosa: prueba de la tuberculina

El diagnóstico de infección ${ }^{3}$ se basa en el viraje tuberculínico (test de Mantoux), que demuestra la existencia de inmunidad celular, cuya positividad aparece entre dos y diez semanas tras la infección.

El cribado tuberculínico se utiliza para identificar, evaluar y poder tratar a las personas que tienen mayor riesgo de padecer una infección tuberculosa latente $o$ de desarrollar la enfermedad tuberculosa una vez ya infectados por el $M$. tuberculosis. Las edades y la frecuencia con que debe efectuarse como cribado sistemático dependen de la prevalencia de la infección tuberculosa en el área o grupo en cuestión. Estará indicado también si existe exposición conocida o probable a TBC y ante clínica o radiología compatibles con TBC.

\section{Intradermorreacción de Mantoux}

Inicialmente, la tuberculina de Koch se obtenía a partir de un cultivo hervido de bacilos tuberculosos. En la actualidad se utiliza un PPD obtenido por filtrado a partir de cultivo de Mycobacterium tuberculosis esterilizado y concentrado. La dosis se mide en unidades de tuberculina (UT). Actualmente, en nuestro medio se usa PPD RT-23 (2 UT/0,1 ml). Anteriormente se utilizó PPD CT-68 (5 UT/0,1 $\mathrm{ml}$ ). Ambas son equivalentes a 5 UT patrón PPD-S (OMS, 1952). Debe conservarse en la oscuridad a $4{ }^{\circ} \mathrm{C}$, evitando mezclarlo con otros viales para evitar confusiones (especialmente con vacunas que se conserven en la misma nevera). El vial debe agitarse antes de extraer cada dosis de tuberculina y esta debe inyectarse antes de 30 minutos después de cargada la jeringa. La solución contiene un estabilizador (Tween 80) que permite utilizar la tuberculina de un envase multidosis hasta agotarlo, mientras no se sobrepase la fecha de caducidad.

Se inyecta 0,1 $\mathrm{ml}$ de solución de PPD, intradérmicamente, en la cara anterior del antebrazo, con jeringa de insulina y aguja del calibre 26 o 27, con bisel corto, orientado hacia arriba. Debe producirse una pápula visible, de 6-10 mm, y no debe sangrar. Se recomienda retrasar algo la retirada de la aguja para minimizar la pérdida de antígeno. Si una dosis se administra incorrectamente, debe repetirse a algunos centímetros de distancia. La reacción alcanza un máximo a las 48-72 horas y puede permanecer algunos días. Debe leerse a las 72 horas (4896) palpando y midiendo el diámetro transversal de la induración que presente, no del eritema, que carece de significación. Se ha de registrar el resultado en milímetros aunque sea $0 \mathrm{~mm}$ (no "posi- 
tivo" o "negativo"). Ha de ser practicada e interpretada por un profesional sanitario entrenado, por lo que no debe confiarse en la lectura por el propio paciente o sus familiares. Se recomienda no cubrir el lugar del pinchazo con un apósito y no rascarse el brazo si existe picor (puede aplicarse una compresa fría). Puede lavarse y secarse normalmente la zona de la punción.

La prueba de la tuberculina carece de contraindicaciones y no produce sensibilización, por lo que su repetición no induce induración en un individuo nunca infectado. Existen falsos positivos y negativos en las circunstancias que se muestran en las tablas 1 y 2 .

Tabla 1. Derivado proteico purificado: causas de falsos negativos

- TBC muy aguda o grave

- Malnutrición

- Inmunodeficiencia (incluido VIH)

- Inmunosupresión

- Neoplasia

- Viriasis (sarampión, varicela...)

- Recién nacidos

- Ancianos

- Dos primeros meses tras la primoinfección

- Almacenamiento incorrecto

- Técnica incorrecta

TBC: tuberculosis; VIH: virus de la inmunodeficiencia humana.
Tabla 2. Derivado proteico purificado: causas de falsos positivos

- Infección por micobacterias atípicas

- Vacunación con BCG previa

- Infección del punto de inyección

- Sangrado en el punto de inyección

- Errores en la preparación

- Errores en la técnica

BCG: bacilo de Calmette-Guérin

Las vacunas vivas atenuadas (sarampión, rubeola, parotiditis, varicela, polio oral, fiebre amarilla, BCG y tifoidea oral) pueden suprimir la respuesta a la tuberculina. Ya que esta supresión no se produce en las 48 horas posteriores a la administración de la vacuna triple vírica, el PPD debe efectuarse simultáneamente a la vacuna o un mes después.

Un PPD negativo no excluye totalmente la TBC. Hasta un $10-20 \%$ de niños con infección tuberculosa demostrada por cultivo no reaccionan inicialmente a la prueba, aunque sí lo hacen, en su mayoría, posteriormente. En las diseminaciones hematógenas este porcentaje de falsos negativos iniciales puede llegar hasta el $50 \%$, por la anergia inducida por la propia infección. En los infectados por VIH depende de su grado de inmunosupresión.

Un inconveniente del PPD es que el derivado proteico no es específico del 
Mycobacterium tuberculosis, lo que reduce la especificidad de la prueba de la tuberculina. Si la prevalencia de infecciones por micobacterias atípicas es alta, parte de las reacciones positivas se deberán a infecciones por esas micobacterias. Los falsos positivos por esta causa no son frecuentes en nuestro país y pocas veces resultarán superiores a 5-10 mm.

Más dudas plantea la posibilidad de falsos positivos por vacuna BCG previa. La positividad del PPD se desvanece con el tiempo y raramente es mayor de $15 \mathrm{~mm}$. Si resulta mayor de $15 \mathrm{~mm}$ o si, aun con resultados menores, existe sospecha clínica de TBC o se conoce la exposición a un caso, debe actuarse según el resultado de la prueba, independientemente del antecedente de BCG. En los vacunados expuestos a un bacilífero el VPP de un resultado de $5 \mathrm{~mm}$ es del $72 \%$, y la prueba debe considerarse positiva. Se considera también infectado si aparece vesiculación o necrosis o si proviene de un país con alta prevalencia de TBC. Solo suelen haber dudas en pacientes asintomáticos, vacunados menos de cinco años antes, sin exposición conocida o probable. En los pacientes vacunados las pruebas in vitro de liberación de interferón-gamma (IGRA) resultan negativas, mientras que los infectados son IGRAt. Ello puede ayudar en la toma de decisiones.

\section{Repetición de la prueba. Conversión tuberculínica. Efecto booster}

No debe repetirse la prueba si existe el antecedente de una prueba positiva anterior, independientemente de su tamaño y del tiempo transcurrido desde entonces, pues el efecto booster puede amplificar el resultado del test y llevar a conclusiones erróneas. La tuberculina no sensibiliza aunque se practique más de una vez, por lo que si resulta positiva en quien anteriormente tenía una prueba negativa, permite suponer que ha existido una infección tuberculosa.

Se considera convertor o viraje tuberculínico reciente el paso en menos de dos años de negativo a positivo o el incremento del diámetro de la induración de $6 \mathrm{~mm}$ o más. Este hecho tiene implicaciones: supone infección también reciente; implica alto riesgo de desarrollar enfermedad TBC; y obliga a buscar individuos bacilíferos entre los contactos.

Con el paso del tiempo, en el infectado de TBC se debilita la respuesta a la tuberculina y el resultado de la prueba puede hacerse negativo. Pero la capacidad de respuesta persiste y el PPD puede actuar como estímulo (efecto booster) y una segunda prueba practicada poco después resultar positiva. Este efecto aparece al menos siete días tras la primera prueba 
considerada como negativa y suele durar un año tras esa prueba, pero puede persistir durante muchos más. Para descartar que la prueba negativa se deba no a ausencia de infección sino a debilitamiento de la respuesta, se practica una segunda prueba 7-10 días después y el resultado de esta segunda prueba será el que se tendrá en consideración. Si es positiva se atribuirá al efecto booster, evitando un falso diagnóstico de convertor en el futuro. Si es negativa se considerará no infectado hasta la fecha y un posible resultado positivo más adelante sí permitirá diagnosticarlo de conversión tuberculínica.

La infección tuberculínica se presenta sobre todo en la infancia o la juventud y el debilitamiento de la respuesta tiene lugar habitualmente en edades avanzadas (> 55 años). Pero como puede presentarse antes, en sujetos en quienes va a tener que repetirse la prueba frecuentemente por su mayor riesgo de contraer o transmitir la TBC (maestros, personal sanitario, etc.), si la primera prueba efectuada es negativa se recomienda repetirla a los 7-10 días y aceptar como definitivo este segundo resultado. Se haría así, por ejemplo, en un profesional sanitario al iniciar su actividad laboral, para evitar posteriormente la confusión con una conversión atribuible a una infección reciente en futuras pruebas.
La sensibilidad tuberculínica por vacunación BCG se debilita más pronto que la debida a la infección natural. Por ello, en vacunados de cualquier edad en que la prueba resulte negativa, la repetición de la prueba a los 7-10 días permitirá comprobar el efecto booster. La infección por micobacterias atípicas se comporta, en este sentido, como la BCG.

\section{Métodos diagnósticos in vitro (IGRA)}

En los últimos años se han investigado métodos diagnósticos basados en la cuantificación de la respuesta inmune celular ${ }^{4}$. Estos métodos, denominados interferon-gamma release assays (IGRA) detectan la liberación de interferóngamma en respuesta a antígenos micobacterianos y miden la respuesta inmune a estos antígenos en sangre periférica. La primera generación de quantiFERON-TB, aprobada por la FDA en 2001, medía la liberación de interferón-gamma en respuesta al PPD. En 2004 se aprobó la segunda generación del test, quantiFERON-TB Gold, que no emplea PPD sino antígenos más específicos, que no se encuentran en las cepas que contiene la BCG ni en la mayoría de micobacterias atípicas. La otra técnica disponible actualmente (T-SPOT.TB), mide el número de células que liberan interferón 
gama. T-SPOT.TB es algo más sensible que la prueba de la tuberculina en el estudio de contactos, mientras que QuantiFERON-TB Gold tiene una sensibilidad similar.

Tienen las ventajas, frente a la prueba de la tuberculina, de no verse afectados por la BCG y la mayoría de micobacterias no tuberculosas, requerir una sola visita, no originar efecto booster, por lo que puede ser preferible cuando se necesiten controles seriados, y ser menos sensibles a los sesgos inherentes a la lectura de la prueba de la tuberculina. Las desventajas principales son la necesidad de una muestra de sangre, que además debe procesarse en pocas horas, y la falta de datos referentes a su empleo en menores de cinco años.

En adultos, para el diagnóstico de la infección TBC la sensibilidad y especificidad de estas pruebas es de más del $90 \%$. Para el diagnóstico de la enfermedad, la sensibilidad es algo menor por la depresión inmunitaria que la propia enfermedad comporta. En niños se dispone de menos estudios. Un test negativo, al igual que un PPD negativo, no excluye con seguridad la presencia de ITL o de enfermedad tuberculosa. Los IGRAs tampoco permiten diferenciar entre ITL y enfermedad tuberculosa activa $y$, al igual que con el Mantoux, son necesarias otras pruebas para poderla descartar o confirmar.

\section{Indicaciones del cribado tuberculínico}

La estrategia debe orientarse a encontrar, para ofrecerles tratamiento, a las personas con infección tuberculosa latente que se encuentren en mayor riesgo para desarrollar la enfermedad. En general, las personas con mayor riesgo para desarrollar la enfermedad tuberculosa son las que tienen una mayor posibilidad de exposición a individuos enfermos de TBC y aquellas cuyas condiciones clínicas u otros factores se asocian a un incremento del riesgo de progresar de infección a enfermedad tuberculosa.

El valor predictivo de una prueba de cribado depende de su sensibilidad y especificidad, teóricamente valores fijos, y de la prevalencia de la enfermedad a detectar. El VPP aumenta con la prevalencia de la enfermedad. Por ello, en poblaciones con baja prevalencia (baja tasa de positividad tuberculínica) no está indicado el cribado sistemático ya que, además de haber pocos positivos, en su mayoría serían falsos positivos. Para aumentar su VPP el test debe practicarse solo en grupos de alto riesgo, con mayor prevalencia que en la población general. Su sensibilidad y especificidad dependerán, por 
otra parte, de donde se establezcan los puntos de corte para considerarlo positivo o negativo.

Se recomienda efectuarlo si hay tasas superiores al $1 \%$ a los seis años. En España, salvo en algunas bolsas de pobreza o población marginal, la prevalencia de la infección tuberculosa a esta edad es inferior a esa cifra y no se recomienda el cribado sistemático. Podría plantearse a los 13-14 años, cuando la prevalencia ya es algo mayor (3\%). Las edades óptimas y la periodicidad con que se practicará dependerán de las circunstancias locales, pero indicaciones generalmente aceptadas son:

- Hijos de adictos a drogas por vía parenteral o de portadores de infección $\mathrm{VIH}$.

- Grupos marginales o de nivel socioeconómico muy bajo.

- Niños con inmunodeficiencias o seropositivos para VIH.

- Previamente a tratamiento inmunosupresor.

- Contacto o sospecha de contacto con enfermo bacilífero.

- Sospecha clínica o radiológica de infección TBC.

- Primera valoración de niños inmigrantes o procedentes de adopción desde países donde la TBC es endémica (Asia, Oriente Medio, África,
Latinoamérica, Europa del Este, Rusia).

- Antes y después de viajar a estos países (estancia superior un mes).

La tabla 3 recoge las indicaciones para la práctica de la prueba de la tuberculina en niños y adolescentes propuestas por el documento de consenso (2009) de la Sociedad Española de Infectología Pediátrica y la Sociedad Española de Neumología Pediátrica.

En estos casos el cribado se hará de manera oportunista, repitiéndose según las posibilidades y las prioridades establecidas localmente. Las recomendaciones varían según la fuente de la que emanan pero todas incluyen:

- Cada 1-2 años en los grupos de riesgo y en zonas urbanas con factores de riesgo.

- Anualmente en infectados por VIH.

- Ciertas edades, según la epidemiología local, pero suele ser al empezar y acabar la escuela primaria, en niños sin factores de riesgo pero que viven en zonas con alta prevalencia de infección TBC.

Los inmigrantes ${ }^{5}$ que llegan a nuestro país proceden mayoritariamente de países de renta baja donde las tasas de infección y enfermedad tuberculosa son elevadas. Por ello, estos niños deben considerarse un grupo de riesgo y está 


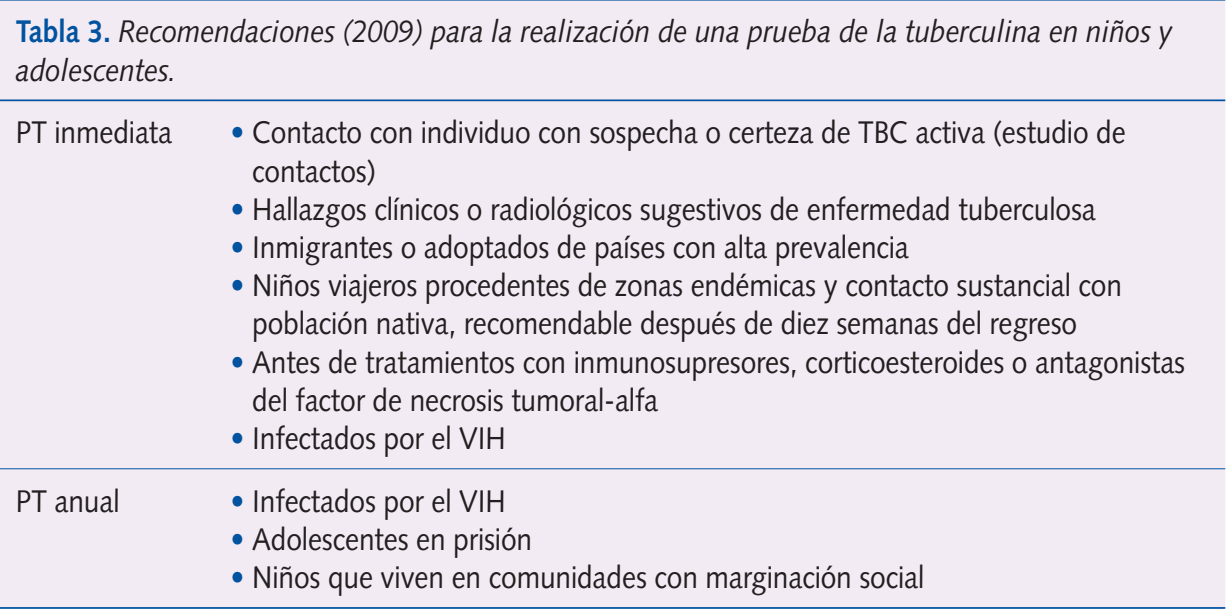

PT: prueba de la tuberculina; TBC: tuberculosis; VIH: virus de la inmunodeficiencia humana.

Fuente: Sociedad Española de Infectología Pediátrica y Sociedad Española de Neumología Pediátrica (2009).

indicado tanto el cribado de la infección como la búsqueda activa de la enfermedad. Se recomienda la prueba de la tuberculina:

- Al contactar por primera vez con el sistema sanitario.

- Cuando se sospeche TBC o haya estado en contacto con un enfermo de TBC.

- Al menos cada dos años durante los primeros cinco años de estancia en nuestro país.

- Cada dos años, indefinidamente, si está en contacto repetidamente con personas que provienen de países en desarrollo, mientras el PPD siga siendo negativo.

Si han sido vacunados con BCG y el PPD es negativo, ya que en este colecti- vo se prevé un seguimiento periódico, se recomienda efectuar una segunda prueba 7-15 días después para ver si la primera provoca un efecto booster. En este caso el diámetro de la segunda prueba será habitualmente superior a $5 \mathrm{~mm}$ sin que suponga una infección reciente y servirá de valor de referencia para pruebas sucesivas.

Aunque no se recomienda la búsqueda activa de casos en niños no pertenecientes a los grupos de riesgo y sin síntomas clínicos sugestivos de TBC, algunos autores recomiendan la práctica de una prueba de tuberculina en los adolescentes de 14-16 años, excluyendo a los que se saben ya positivos, ya que a esta edad el riesgo de infección se eleva significativamente. Se considera que así podría de- 
tectarse el $80 \%$ de la población infectada. En la mayoría de los adolescentes resultará negativa pero les servirá de referencia para el diagnóstico de cuadros clínicos compatibles con TBC que puedan aparecer posteriormente.

\section{Interpretación del PPD.}

\section{Recomendaciones de distintos grupos de expertos}

Las recomendaciones de los distintos grupos de expertos difieren según el entorno en que se efectúan esas recomendaciones. Los principales factores a considerar al establecer los criterios de positividad para la prueba de la tuberculina son:

- La prevalencia de la infección tuberculosa en la población de referencia.

- La prevalencia de la infección por micobacterias atípicas en esa misma población.

- El antecedente de vacunación BCG en el sujeto al que se efectúa la prueba.

- El riesgo del sujeto para contraer la infección y para desarrollar la enfermedad tuberculosa si la contrae.

Si la prevalencia de infección tuberculosa en la población o grupo al que pertenece el sujeto es baja, la mayoría de pruebas positivas corresponderán a falsos positivos. Por el contrario, cuanto mayor sea la prevalencia, más pruebas positivas lo serán por una verdadera infección. Si la prevalencia de infecciones por micobacterias atípicas es alta, parte de las reacciones positivas a la prueba de la tuberculina se deberán a infecciones por micobacterias atípicas.

En función de la sensibilidad y especificidad de la prueba de tuberculina y de la prevalencia de tuberculosis en los distintos grupos de población, los CDC (EE. UU.) establecen tres puntos de corte para definir una reacción positiva a la tuberculina:

- $5 \mathrm{~mm}$ : personas con alto riesgo de desarrollar enfermedad tuberculosa si se infectan: contactos recientes con TBC, radiografía sugestiva, inmunodeprimidos.

- $10 \mathrm{~mm}$ : personas con inmunidad normal, con alta posibilidad de infectarse pero sin otros factores de riesgo.

- 15 mm: personas con poca posibilidad de infectarse: cribado en institutos, empresas.

La American Academy of Pediatrics considera el PPD positivo en los siguientes casos $^{6}$ :

- $5 \mathrm{~mm}$ : niños en contacto íntimo con un caso índice o sospechoso de TBC, niños con sospecha clínica o radiológica de TBC, niños con inmunodepresión o infección por VIH. 
- 10 mm: niños con mayor riesgo de TBC diseminada (menores de cuatro años o con malnutrición, linfoma, diabetes, insuficiencia renal crónica), nacidos o hijos de padres nacidos en países con alta prevalencia de TBC, niños con exposición frecuente a adultos de riesgo para $\mathrm{TBC}(\mathrm{VIH}$, $A D V P$, vagabundos, personal de instituciones o institucionalizados, inmigrantes, personal de granjas...) y virajes recientes (dos años).

- $15 \mathrm{~mm}$ : niños mayores de cuatro años sin ningún contacto ni factor de riesgo.

El documento 7 de consenso (2009) de la Sociedad Española de Infectología Pediátrica y la Sociedad Española de Neumología Pediátrica establece dos puntos de corte de positividad de la prueba de la tuberculina, situando el dintel en un menor tamaño $(5 \mathrm{~mm})$ para niños con riesgo elevado de desarrollar la enfermedad y un mayor tamaño $(10 \mathrm{~mm})$ para niños en situación de menor riesgo (tabla 4). En relación al diagnóstico de la ITL mediante Quantiferon Gold y T-SPOT-TB establece las siguientes recomendaciones:

- La prueba de la tuberculina es aún el test de elección en el diagnóstico de la ITL.

- Los IGRA se utilizan como prueba complementaria para incrementar la
Tabla 4. Criterios de positividad (2009) de la prueba de la tuberculina

\section{$5 \mathrm{~mm}$}

- Niños en contacto íntimo con un caso índice o sospechoso de tuberculosis

- Niños sospechosos de enfermedad tuberculosa clínica o radiológica

- Niños en situaciones de inmunodepresión o infección por VIH

- Niños con conversión de la prueba de tuberculina previamente negativa

\section{$10 \mathrm{~mm}$}

- Cualquier otro caso, incluido el niño inmigrante, viajero y el cribado de niños sanos, independientemente de existir antecedentes de vacunación con BCG

BCG: bacilo de Calmette-Guérin; VIH: virus de la inmunodeficiencia humana.

Fuente: Sociedad Española de Infectología Pediátrica y Sociedad Española de Neumología Pediátrica (2009).

especificidad reduciendo la interferencia por la BCG o la infección por micobacterias atípicas.

- En el estudio de contactos, Ios IGRA deben ser utilizadas para el diagnóstico de la ITL en función del riesgo de ITL y de la existencia de inmunosupresión.

\section{Prevención de la tuberculosis}

Las medidas preventivas ${ }^{8-9}$ en relación a la TBC incluyen:

- Evitar el contacto con individuos con infección activa. 
- Quimioprofilaxis.

- Vacuna BCG en la población de alto riesgo.

- Mejorar las condiciones socioeconómicas y sanitarias.

La disminución de la exposición a la TBC se lograría reduciendo el número de enfermos contagiosos mediante la búsqueda activa de infectados y enfermos en los grupos de riesgo y la sospecha clínica en individuos de la población general. En ambos casos se efectuará el diagnóstico y tratamiento adecuados y se aislarán mientras sean bacilíferos. La TBC es una enfermedad de declaración obligatoria individualizada y nominal.

\section{Grupos de riesgo}

La detección y tratamiento de la ITL son importantes en el control de la TBC, pero el cribado universal no es eficiente y no se recomienda su práctica sistemática, debiendo reservarse para grupos de riesgo. En nuestro entorno constituyen grupos de riesgo tributarios de especial énfasis en la sospecha clínica y de controles periódicos de tuberculina:

- Infectados por VIH o sujetos de riesgo para esta infección.

- Contactos íntimos y/o frecuentes con bacilíferos, sobre todo de reciente diagnóstico.
- Convertores tuberculínicos recientes.

- Minorías étnicas en situación socioeconómica precaria.

- Individuos con inmunodeficiencia o inmunosupresión.

- Sujetos institucionalizados: prisiones, asilos, instituciones.

- Sujetos que por su profesión tienen mayor riesgo de adquirir la TBC o de transmitirla si la padecen: sanitarios, personal de guarderías o escuelas, etc.

Arbitrariamente, se consideran grupos o poblaciones de riesgo aquellos en los que la incidencia de casos de TBC es mayor de 100/100 000 o netamente superior a la propia de su comunidad. En el caso de los niños, se incluyen en los grupos de alto riesgo:

- Hijos o contactos frecuentes de enfermos de TBC, especialmente si son bacilíferos, enfermos crónicos o mal cumplidores.

- Hijos o contactos frecuentes de infectados por $\mathrm{VIH}$.

- Hijos o contactos frecuentes de presidiarios, expresidiarios recientes (dos años), indigentes o individuos con sociopatías importantes.

- Hijos o contactos frecuentes de inmigrantes recientes (dos años) de países con alta prevalencia de infección TBC. 
- Residentes en zonas geográficas de alta endemicidad.

- Residentes en zonas sociosanitariamente deprimidas.

- Niños inmunodeprimidos por alguna enfermedad de base o su tratamiento.

En los niños de estos grupos de riesgo se aconseja:

- Efectuar un PPD si presentaron una prueba negativa hasta 1-2 años antes o si se desconoce su situación en relación a la infección TBC (nunca se ha realizado un PPD o se desconoce su resultado).

- Estudio radiológico a todos los reactores de $\geq 5 \mathrm{~mm}$, independientemente de sus antecedentes de vacunación BCG.

- Indicar tratamiento de la enfermedad tuberculosa o de la infección probable o de la infección latente, si procede.

- Esta sistemática se iniciará a partir de los 1-2 años de vida y, si las circunstancias epidemiológicas no cambian, cada 1-2 años, repitiéndose el PPD si era negativo.

- Si ya era positivo y recibió tratamiento o profilaxis se efectuará una anamnesis y una exploración adecuada y radiología de tórax si hay signos o síntomas sospechosos.

\section{Estudio de contactos}

El estudio de contactos ${ }^{10-12}$ a partir de un caso índice tiene como objetivos:

- Diagnosticar a enfermos o infectados (casos secundarios).

- Tratar precozmente a los enfermos e infectados que lo precisen.

- Rehacer la cadena de transmisión para identificar, si se puede, el caso inicial.

El diagnóstico de una infección tuberculosa en un niño obliga a buscar otros casos entre sus contactos ya que hasta el $50 \%$ de contactos íntimos de un bacilífero pueden estar infectados. Deben estudiarse los contactos de todas las formas de TBC, pulmonares o extrapulmonares, siendo prioritarios los contactos de enfermos con baciloscopia positiva (máxima prioridad), menores de 15 años PPD+; y convertores recientes.

Prioridades en el estudio de contactos:

- Alta prioridad: personas con contacto estrecho o prolongado (más de seis horas al día), niños menores de cinco años y personas con alteración del sistema inmunitario.

- Prioridad mediana: personas con contacto diario, pero menor de seis horas.

- Baja prioridad: contacto esporádico (no diario).

La pauta de estudio (figura 1) es la siguiente: 
- Se inicia con la práctica de PPD a los convivientes y personas con relación estrecha (maestros y cuidadores), excepto si ya se sabía que eran PPD+.

- Se efectúa radiografía de tórax a los contactos íntimos (contacto más de 6 horas diarias) del caso índice, sean PPD+ o PPD-. En otros contactos (frecuentes o esporádicos) la exploración radiológica puede limitarse a quienes resulten ser PPD+.

- Según la edad, el resultado del PPD y la radiología, si se practica, se establece tratamiento de la infección probable o de la infección latente, tratamiento específico o nada. Si van surgiendo PPD+ debe ir ampliándose el círculo de contactos a estudiar.

- En los contactos de un bacilífero en quienes la tuberculina resulte negativa se repetirá la prueba al cabo de 8-12 semanas para descartar un contagio muy reciente.

- Todos los contactos de un enfermo de TBC que no reciban quimioprofilaxis o quimioterapia deben controlarse de nuevo a los pocos meses.

En las TBC primarias, propias de niños y jóvenes, el diagnóstico bacteriológico es más difícil al ser lesiones cerradas, con escasa población bacilar. En estos casos, el antecedente de contacto con un tu- berculoso, si existe clínica o radiología sugestiva y el PPD positivo, bastan para el diagnóstico. En niños pequeños, el TC torácico ${ }^{13}$ puede resultar de ayuda.

\section{Tratamiento de la infección proba- ble (TIP) o prevención de la infección}

Antes llamado quimioprofilaxis primaria, pretende prevenir la infección en un individuo no infectado y por ello PPD-, expuesto de forma estrecha o continuada a un bacilífero ${ }^{14}$. Está indicado en sujetos PPD- de cualquier edad y resulta obligado en los niños. En los menores de cinco años, con máximo riesgo de infectarse tras la exposición, se recomienda además la separación del foco TBC durante las primeras semanas de profilaxis.

Se administra isoniazida a $5 \mathrm{mg} / \mathrm{kg} /$ día, máximo 300 mg/día, en dosis única por la mañana en ayunas, hasta 2-3 meses tras cesar el contacto con el enfermo o dejar este de ser contagioso. Se repite el PPD en ese momento, suspendiéndose la isoniazida si cumple todas estas condiciones:

- El segundo PPD es negativo

- El niño tiene más de seis meses (los infectados pueden ser anérgicos hasta esa edad).

- La segunda prueba se ha efectuado al menos diez semanas después de la primera. 
Si el PPD se ha positivizado y la radiología y la clínica permiten descartar enfermedad TBC, se continuará como en el tratamiento de la infección tuberculosa latente. Si la clínica o la radiología sugieren enfermedad TBC se tratará según las pautas de quimioterapia establecidas para ello (figura 1).

\section{Tratamiento de la infección tuberculosa latente (TIL)}

Antes llamado quimioprofilaxis secundaria, pretende prevenir la aparición de enfermedad en un sujeto infectado, PPD+, tras descartar la presencia de enfermedad activa ${ }^{15-16}$. Su eficacia es supe- rior al $90 \%$ en la prevención de la enfermedad TBC. El máximo riesgo de pasar de infectado a enfermo se da en niños, adolescentes, infectados recientemente y personas con depresión de la inmunidad celular, por lo que sus indicaciones incluyen: - En niños, siempre que presenten PPD+, tras descartar enfermedad TBC.

- En otras edades, en función del riesgo de enfermar:

- PPD+ hasta 20 años.

- PPD+ hasta 35 años si tienen contacto estrecho con un bacilífero o factores de riesgo, sociales o profesionales.

Figura 1. Pauta de actuación ante el contacto con un enfermo o infectado de tuberculosis.

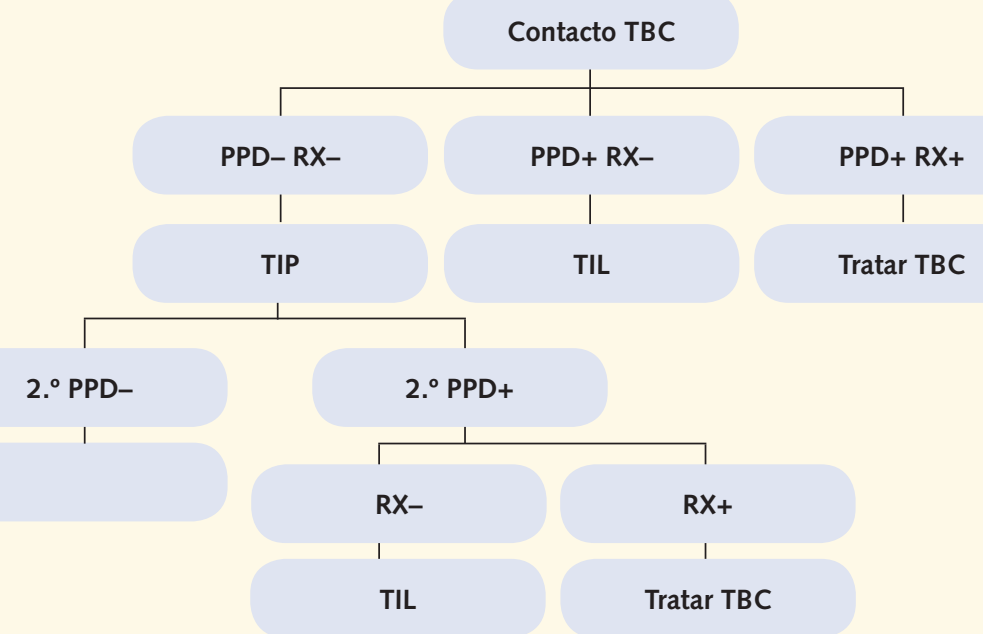

PPD: prueba de la tuberculina; RX: radiografía de tórax; TIL: tratamiento de la infección latente; TIP: tratamiento de la infección probable. 
- Convertores recientes (últimos dos años).

- Infectados por VIH o sujetos de riesgo para esta infección (UDVP).

- Patología de base con riesgo aumentado de desarrollar enfermedad TBC.

Se usa también isoniazida, $5 \mathrm{mg} / \mathrm{kg} /$ día hasta $300 \mathrm{mg} /$ día, con una duración óptima de nueve meses (recomendada en niños), aunque con seis meses se consigue una protección aceptable (69\%) y se facilita el cumplimiento. En niños con factores de riesgo (familiares bacilíferos, inmunodeprimidos o virajes recientes) debe mantenerse nueve meses. En infectados por $\mathrm{VIH}$, hasta 12 meses. No tiene ventajas alargarlo más de 12 meses, excepto en niños PPD+ que tras completar el TIL reciban corticoides o inmunosupresores de forma prolongada, en cuyo caso se mantendrá hasta terminar ese tratamiento. El TIL no está indicado en individuos PPD+ ya tratados con tuberculostáticos, aun de forma incorrecta o incompleta, sea por infección o por enfermedad TBC.

Antes de comenzar debe asegurarse no estar ya ante un enfermo. No es una situación urgente y deben completarse previamente los estudios pertinentes. Existe a veces la duda de si se está tratando con monoterapia una enfermedad tuberculosa inadvertida, sobre todo en formas extrapulmonares, niños pequeños o inmunodeprimidos. Se están estudiando, por ello, asociaciones de fármacos, como la pauta de seis meses de isoniazida y rifampicina a las dosis habituales en un solo preparado. Algunos autores proponen profilaxis con isoniazida y rifampicina en menores de cinco años con contagio doméstico y PPD+, especialmente si este es mayor de $12 \mathrm{~mm}$ o existe vesiculación o necrosis, por el alto riesgo de diseminación, las dificultades diagnósticas propias de esa edad y el peligro de una monoterapia inadvertida. Ambos fármacos se mantendrían al menos 6-8 semanas, tiempo en el que se minimizaría la población bacilar. Después, si ni la clínica ni la radiología son sugestivas de enfermedad TBC, se seguiría con solo isoniazida.

Los niños de pocos años PPD+ es posible que tengan ya la enfermedad aunque se encuentren asintomáticos y con radiología de tórax normal. En este grupo de niños algunos autores recomiendan un $\mathrm{TC}$ torácico ${ }^{13}$. No existe consenso y debe considerarse la radiación que supone el TC y la probable necesidad de sedación. Los efectos adversos del TIL con isoniazida, principalmente hepatotoxicidad, son infrecuentes. En el $10-20 \%$ de pacientes que la reciben aparecen elevacio- 
nes asintomáticas de las transaminasas. Es aceptable un aumento de hasta cinco veces los valores normales en pacientes sin síntomas clínicos de hepatitis, siempre que la bilirrubina sérica permanezca normal. Se presenta hepatitis clínica en el $0,1 \%$ de pacientes tratados con isoniazida. El riesgo aumenta con la edad, la presencia de hepatopatía previa y el consumo de alcohol, por lo que la TIL se restringe a partir de los 35 años salvo en infectados por $\mathrm{VIH}$, en quienes los bene- ficios superan el riesgo. Los niños no precisan pruebas hepáticas ni antes ni durante el TIL, salvo hepatopatía previa conocida o signos de toxicidad.

Es importante el control regular en la consulta, interrogando sobre el cumplimiento, toxicidad o clínica de TBC, y reforzando la importancia de completar el tratamiento. No es preciso repetir la radiografía si la efectuada al iniciar el tratamiento fue normal y el niño sigue asintomático.

\section{Bibliografía}

1. Control mundial de la tuberculosis-Informe OMS 2011. [Internet]. [Consultado el 28/11/2011]. Disponible en www.who.int/tb/ publications/global_report/es/

2. Ministerio de Sanidad y Consumo. Plan para la prevención y control de la tuberculosis en España. 2008 [en línea] [consultado el 28/11/2011]. Disponible en www.msc.es/profesionales/salud Publica/prevPromocion/planTuberculosis.htm

3. Gómez-Pastrana D. Diagnóstico de la tuberculosis pulmonar. An Pediatr (Barc). 2007;66(Supl 2):45-51.

4. CDC. Updated guidelines for using interferon gamma release assays to detect Mycobacterium tuberculosis infection-United States 2010. MMWR. 2010;59(RR05):1-25.

5. Masvidal RM, Gil BM, Vall M, Zabaleta E, Carnero E, Rodríguez de la Rubia C. Estudio de infección tuberculosa en una zona de gran incidencia de tuberculosis y con un elevado porcentaje de inmigrantes. An Pediatr (Barc). 2004;60:22-7.
6. Pediatric Tuberculosis Collaborative Group. Targeted tuberculin skin testing and treatment of latent tuberculosis infection in children and adolescents. Pediatrics. 2004;114:1175-201.

7. Diagnóstico de la tuberculosis en la edad pediátrica. Documento de consenso de la Sociedad Española de Infectología Pediátrica (SEIP) y la Sociedad Española de Neumología Pediátrica (SENP). An Pediatr (Barc). 2010;73:143.e1-143. e14.

8. Carceller A, Lebel M. Prevención de la tuberculosis en España en el siglo XXI. An Pediatr (Barc). 2005;62:207-9.

9. Altet $\mathrm{N}$, Alcaide J. Control y eliminación de la tuberculosis en España: las estrategias para el siglo XXI. An Pediatr (Barc). 2006;64:66-73.

10. . Documento de consenso sobre el estudio de contactos en los pacientes tuberculosos. Grupo de Estudio de Contactos de la Unidad de Investigación en Tuberculosis de Barcelona (UITB). Med Clin (Barc). 1999;112:151-6.

11. CDC. Guidelines for the investigation of contacts of persons with infectious tuberculosis: 
recommendations from the National Tuberculosis Controllers Association and CDC. MMWR. 2005;54(No.RR-15):1-48.

12. Salinas C, Ballaz A, Diez R, Iza de Pablo J, Pocheville I, Aguirre U. Study of contacts in children and adolescents using the QuantiFERON ${ }^{{ }_{-}}$ TB Gold in-Tube. An Pediatr (Barc). 2011;74:36370.

13. Gómez-Pastrana D, Carceller-Blanchard A. ¿Debe realizarse una tomografía computarizada torácica a los niños con infección tuberculosa sin enfermedad aparente? An Pediatr (Barc). 2007;67: 585-93.
14. Grupo de trabajo de la Sociedad Española de Infectología Pediátrica. Documento de consenso sobre el tratamiento de la exposición a tuberculosis y de la infección tuberculosa latente en niños. An Pediatr (Barc). 2006;64:59-65.

15. Nelson LJ, Jereb JA, Castro KG. New guidelines about latent tuberculosis infection in children and adolescents: A welcome advancement. Pediatrics. 2004;114:1084-6.

16. CDC. Latent Tuberculosis Infection: A Guide for Primary Health Care Providers [en línea] [consultado el 28/11/2011]. Disponible en www. cdc.gov/tb/publications/LTBI/default.htm 